\title{
Exact Solution of the Master Equation for the Asymmetric Exclusion Process
}

\author{
Gunter M. Schütz \\ Institut für Festkörperforschung, \\ Forschungszentrum Jülich, 52425 Jülich, Germany \\ e-mail: g.schuetz@kfa-juelich.de
}

\begin{abstract}
Using the Bethe ansatz, we obtain the exact solution of the master equation for the totally asymmetric exclusion process on an infinite one-dimensional lattice. We derive explicit expressions for the conditional probabilities $P\left(x_{1}, \ldots, x_{N} ; t \mid y_{1}, \ldots, y_{N} ; 0\right)$ of finding $N$ particles on lattice sites $x_{1}, \ldots, x_{N}$ at time $t$ with initial occupation $y_{1}, \ldots, y_{N}$ at time $t=0$.
\end{abstract}

Key words: Asymmetric exclusion process, Bethe ansatz

PACS numbers: $05.40+\mathrm{j}, 02.50 \mathrm{Ga}, 05.70 . \mathrm{Ln}$ 


\section{Introduction}

Driven lattice gases and particularly the one-dimensional asymmetric simple exclusion process (ASEP) have been intensively studied over the past decade for a variety of reasons 回. The ASEP (for a definition see below) has been suggested already in 1968 as a model for the kinetics of biopolymerization [2]. Two years later this process was introduced into the mathematical literature [3] where it has received considerable attention in the context of interacting

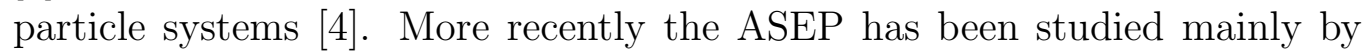
physicists as a model for polymers in random media and as a dynamical model for interface growth [5]. It is also a discrete version of the noisy Burgers equation [6] and thus of interest for the study of shocks [7, 8] and for traffic models [9]. While there are exact solutions and a good understanding of the stationary behaviour of the system with (trivial) periodic and (nontrivial) open boundary conditions with injection and absorption of particles [10] - [13], exact results for the dynamics of the model are scarce (see e.g. Refs. [4, 14] and, for more recent work, Refs. [8, 15, 16] and references therein. It is the aim of this paper to present a new approach to this open problem by explicitly solving the master equation for the system defined on an infinite lattice.

We study the ASEP with sequential updating. In the totally asymmetric version of this very simple model each lattice site can be occupied by at most one particle and particles hop with rate 1 to their right neighbouring site if this was empty. If it was occupied, the attempted move is rejected. This defines a Markov process with state space $X=\{0,1\}^{S}$ where a given configuration $\underline{n} \in X$ is the set of occupation numbers $n_{k}=0,1$ with the site label $k \in S$. Alternatively, if one restricts oneselves to studying the system with an arbitrary, but finite number of particles, one may define the process on $Y=\left\{\{\emptyset\},\left\{k_{1}\right\},\left\{k_{1}, k_{2}\right\}, \ldots,\right\}$ which is the collection of all finite subsets of $S$. In this case one identifies a configuration $\underline{n}$ which has particles on sites $k_{1}, \ldots, k_{N}$ with the corresponding set in $Y$. Here we consider the system with finitely many particles $N$ defined on an infinite one-dimensional lattice $S=\mathbb{Z}$

A convenient presentation of the ASEP is in terms of a master equation for the probability $P\left(B_{N} ; t\right)$ of finding $N$ particles on sites $B_{N}=\left\{k_{1}, \ldots, k_{N}\right\} \in$ $Y$ at time $t$. Defining the ASEP alternatively in terms of a master equation on the state space $X$ rather than $Y$ has turned out to be useful in previous work. In this case the stochastic time evolution is manifestly seen to be generated by the quantum Hamiltonian of a spin- $1 / 2$ Heisenberg chain. This suggests the use of the Bethe ansatz and the quantum group symmetry for the calculation of energy gaps (which give e.g. the dynamical exponent of the system) [15, 18] and certain time-dependent correlation functions [17, 19]. For the purposes of this paper using the coordinate representation $Y$ of the 
state space is more transparent, as in this case the master equation can be solved directly and explicitly using the coordinate Bethe ansatz [20, 21]. While here we consider mainly the totally asymmetric case, the partially asymmetric exclusion process may be solved in the same way. We discuss this for the two-particle problem.

In fact, even though the two-particle system may seem trivial, it exhibits already some of the characteristic behaviour of the driven system at finite particle density. In the undriven system (i.e. in the symmetric exclusion process) the diffusive spreading of a local perturbation does not depend on the overall density. In contrast, in the asymmetric case the center of mass of a local perturbation in an otherwise homogeneous finite background density is, from numerical work, known to spread superdiffusively [1]. This implies a divergent collective diffusion constant as the number of particles tends to infinity. There are, however, no quantitative results for a system with a small number of particles. As we will show here, in a system of two particles this behaviour appears as an increase of the (collective) diffusion constant which turns out to be the single-particle diffusion constant plus a term proportional to the square of the asymmetry in the hopping rates.

The paper is organized as follows: Sec. 2 gives the main result of the paper. We formulate the master equation and present a (non-constructive) proof that the expression derived from the Bethe ansatz in Sec. 3 is indeed the solution. This is done because the proof is elementary and no reference to the Bethe ansatz is necessary. A reader not interested in the derivation of the solution may therefore skip Sec. 3 where the solution is constructed. As a simple application, Sections 4 and 5 focus on properties of the twoparticle system. In Sec. 4 we briefly study the partially asymmetric process where particles are allowed to move both to the right and to the left, but with different rates. We restrict ourselves to the exact solution for the twoparticle system, but also explain how to obtain the solution for the general $N$-particle case. In Sec. 5 we obtain a very simple new result, which shows that already in the two-particle system the diffusive behaviour of the driven system is substantially different from the symmetric, undriven process. In Sec. 6 we present our conclusions.

\section{Solution of the master equation}

Let $P\left(B_{N} ; t\right)$ be the probability of finding $N$ particles on the set of sites $B_{N}=\left\{k_{1}, \ldots, k_{N}\right\}$ at time $t$. When considering the probability $P$ as a function of the coordinates $k_{i}$ we always assume this set to be ordered, $k_{i}<$ $k_{i+1} \forall i$. It is important to note that as a function of its arguments $k_{i}$ the function $P$ is well-defined in $\mathbb{Z}^{N}$, i.e. also for e.g. $k_{i}=k_{i+1}$ or $k_{i}>k_{i+1}$. However in this domain $P$ is not a probability. In other words, in the domain 
$\Omega_{N}=k_{1}<k_{2}<\ldots<k_{N} \subset \mathbb{Z}^{N}$, the function $P$ is the probability defined above, whereas in $\mathbb{Z}^{N} \backslash \Omega_{N}$ it is defined by the master equation below, but is not a probability.

For the totally asymmetric exclusion process as described in the introduction, $P\left(B_{N} ; t\right)$ defined on $\mathbb{Z}^{N} \times[0, \infty)$ satisfies the master equation

$\frac{d}{d t} P\left(k_{1}, \ldots, k_{N} ; t\right)=P\left(k_{1}-1, \ldots, k_{N} ; t\right)+\ldots+P\left(k_{1}, \ldots, k_{N}-1 ; t\right)-N P\left(k_{1}, \ldots, k_{N} ; t\right)$.

This has to be supplemented by boundary conditions in $\mathbb{Z}^{N}$. If any two neighbouring arguments $k_{i}, k_{i+1}$ are equal, $P$ satisfies

$P\left(k_{1}, \ldots, k_{i}, k_{i+1}=k_{i}, \ldots, k_{N} ; t\right)=P\left(k_{1}, \ldots, k_{i}, k_{i+1}=k_{i}+1, \ldots, k_{N} ; t\right) \forall t \geq 0$.

This boundary condition expresses the exclusion interaction. This is easy to see in the simplest case of two particles. Then (2.1) and (2.2) defined on $\mathbb{Z}^{2}$ read

$$
\frac{d}{d t} P\left(k_{1}, k_{2} ; t\right)=P\left(k_{1}-1, k_{2} ; t\right)+P\left(k_{1}, k_{2}-1 ; t\right)-2 P\left(k_{1}, k_{2} ; t\right)
$$

and

$$
P(k, k ; t)=P(k, k+1 ; t) \quad \forall k \text { and } t \geq 0
$$

which is equivalent to the following equations with $P$ restricted to $\Omega_{2}$

$\frac{d}{d t} P\left(k_{1}, k_{2} ; t\right)=P\left(k_{1}-1, k_{2} ; t\right)+P\left(k_{1}, k_{2}-1 ; t\right)-2 P\left(k_{1}, k_{2} ; t\right)$ if $k_{2}-k_{1}>1$

and

$$
\frac{d}{d t} P\left(k_{1}, k_{2} ; t\right)=P\left(k_{1}-1, k_{2} ; t\right)-P\left(k_{1}, k_{2} ; t\right) \text { if } k_{2}-k_{1}=1 .
$$

The second equation (2.6) expresses that due to exclusion the configuration $(k, k+1)$ can be reached in a single step only from the configuration $k-1, k+1$ and be left only in a single way (which is by moving to $(k, k+2))$. Extending the range of validity of (2.5) to all $\mathbb{Z}^{2}$ requires adding $P\left(k_{1}, k_{2}-1 ; t\right)-$ $P\left(k_{1}, k_{2} ; t\right)$ to (2.6). However, by demanding that (2.4) holds for all times, this is equivalent to adding 0 . Thus (2.5) and (2.6) remain unchanged, i.e., the two sets of equations have the same solutions in the "physical" domain $\Omega_{2}$. It may seem more natural to use the second formulation of the master equation which is in the $N$-particle case the restriction of the validity of 
the master equation (2.1) to the domain $\Omega_{N}$. One could then obtain a set of equations equivalent to (2.1), (2.2) by replacing the boundary condition (2.2) by including appropriately chosen Kronecker delta-functions in (2.1). However, it turns out that solving the equation is more straightforward and transparent in the formulation (2.1), (2.2).

We finally note that with specified initial condition $A_{N} \equiv\left\{l_{1}, \ldots, l_{N}\right\} \in$ $Y$, i.e.,

$$
P\left(B_{N} ; 0\right)=\delta_{A_{N}, B_{N}}
$$

the probability $P\left(B_{N} ; t\right)$ becomes the conditional probability $P\left(B_{N} ; t \mid A_{N} ; 0\right)$ and thus a complete solution of the problem. Also as a function of the arguments $l_{i}$ the function $P$ is a probability only in the domain $l_{1}<\ldots<l_{N}$.

Let us now introduce the function

$$
F_{p}(n ; t) \equiv e^{-t} \sum_{k=0}^{\infty}\left(\begin{array}{c}
k+p-1 \\
p-1
\end{array}\right) \frac{t^{k+n}}{(k+n) !}
$$

where the binomial coefficient and the factorial are defined by the $\Gamma$-function, i.e. $a ! \equiv \Gamma(a+1)$ and

$$
\left(\begin{array}{l}
a \\
b
\end{array}\right) \equiv \frac{\Gamma(a+1)}{\Gamma(b+1) \Gamma(a-b+1)} .
$$

In what follows we shall need only $p, n \in Z$ and $t \in[0, \infty)$. We list some of the properties of $F_{p}(n ; t)$ :

(1) For integer $p \leq 0, F_{p}$ reduces to a finite sum,

$$
F_{p}(n ; t)=e^{-t} \sum_{k=0}^{|p|}(-1)^{k}\left(\begin{array}{c}
|p| \\
k
\end{array}\right) \frac{t^{k+n}}{(k+n) !}
$$

In particular,

$$
F_{0}(n ; t)=\frac{t^{n}}{n !} e^{-t}
$$

(2) For the time derivative one finds

$$
\frac{d}{d t} F_{p}(n ; t)=F_{p-1}(n-1 ; t)=F_{p}(n-1 ; t)-F_{p}(n ; t),
$$


(3) and for the time integral one gets

$$
\int_{0}^{t} d t F_{p}(n ; t)=F_{p+1}(n+1 ; t)-\left(\begin{array}{c}
-n-1+p \\
p
\end{array}\right)=\sum_{k=n+1}^{\infty} F_{p}(k ; t)-\left(\begin{array}{c}
-n-1+p \\
p
\end{array}\right)
$$

(4) At time $t=0$ one has

$$
\lim _{t \rightarrow 0} F_{p}(n ; t)=\left(\begin{array}{c}
-n+p-1 \\
p-1
\end{array}\right)
$$

which vanishes for $n>0$. Now we state the main result of this paper:

Theorem: Let $F\left(B_{N}, A_{N} ; t\right)$ be the $N \times N$ matrix with matrix elements $F_{i j}=F_{i-j}\left(k_{i}-l_{j} ; t\right)$. Then

$$
P\left(B_{N} ; t \mid A_{N} ; 0\right)=\operatorname{det} F\left(B_{N}, A_{N} ; t\right)
$$

is the solution of the master equation (2.1) with boundary condition (2.2) and with initial condition (2.7).

The theorem states that the conditional probability of finding $N$ particles at time $t$ on $B_{N} \subset \Omega_{N}$ if initially (at time $t=0$ ) they had been on sites $A_{N} \subset \Omega_{N}$ is given by the determinant (2.15). How this result was derived is explained in the next section. Here we give a proof of the theorem which is independent of this construction. First we show that the determinant is a solution to the master equation (2.1). Then we show that it satisfies the boundary condition (2.2) and finally we prove that it satisfies the initial condition (2.7).

Proof: (i) Because of the factor $e^{-t}$ in the functions $F_{p}(n ; t)$ the matrix $F$ may be written $e^{-t} \tilde{F}$ and one gets

$$
\frac{d}{d t} \operatorname{det} F=e^{-N t} \frac{d}{d t} \operatorname{det} \tilde{F}-N \operatorname{det} F \text {. }
$$

This accounts for the term $-N P$ on the r.h.s. of (2.1). The time derivative 
of the determinant of $\tilde{F}$ may be written

$$
\begin{aligned}
\frac{d}{d t}\left|\begin{array}{llll}
\tilde{F}_{11} & \tilde{F}_{12} & \ldots & \tilde{F}_{1 N} \\
\tilde{F}_{21} & \tilde{F}_{22} & \ldots & \tilde{F}_{2 N} \\
\vdots & \vdots & \vdots & \vdots \\
\tilde{F}_{N 1} & \tilde{F}_{N 2} & \ldots & \tilde{F}_{N N}
\end{array}\right|= & \left|\begin{array}{llll}
\dot{\tilde{F}}_{11} & \dot{\tilde{F}}_{12} & \ldots & \dot{\tilde{F}}_{1 N} \\
\tilde{F}_{21} & \tilde{F}_{22} & \ldots & \tilde{F}_{2 N} \\
\vdots & \vdots & \vdots & \vdots \\
\tilde{F}_{N 1} & \tilde{F}_{N 2} & \ldots & \tilde{F}_{N N}
\end{array}\right| \\
& +\left|\begin{array}{llll}
\tilde{F}_{11} & \tilde{F}_{12} & \ldots & \tilde{F}_{1 N} \\
\dot{\tilde{F}}_{21} & \dot{\tilde{F}}_{22} & \ldots & \tilde{\tilde{F}}_{2 N} \\
\vdots & \vdots & \vdots & \vdots \\
\tilde{F}_{N 1} & \tilde{F}_{N 2} & \ldots & \tilde{F}_{N N}
\end{array}\right| \\
& +\ldots+1 \\
& +\left|\begin{array}{llll}
\tilde{F}_{11} & \tilde{F}_{12} & \ldots & \tilde{F}_{1 N} \\
\tilde{F}_{21} & \tilde{F}_{22} & \ldots & \tilde{F}_{2 N} \\
\vdots & \vdots & \vdots & \vdots \\
\dot{\tilde{F}}_{N 1} & \tilde{\tilde{F}}_{N 2} & \ldots & \tilde{\tilde{F}}_{N N}
\end{array}\right|
\end{aligned}
$$

The matrix elements in row $i$ are $\left(\tilde{F}_{i-1}\left(k_{i}-l_{1}\right), \tilde{F}_{i-2}\left(k_{i}-l_{2}\right), \ldots, \tilde{F}_{i-N}\left(k_{i}-l_{N}\right)\right)$ and their time derivatives are $\left(\tilde{F}_{i-1}\left(k_{i}-1-l_{1}\right), \tilde{F}_{i-2}\left(k_{i}-1-l_{2}\right), \ldots, \tilde{F}_{i-N}\left(k_{i}-\right.\right.$ $\left.1-l_{N}\right)$ ), see (2.12). Thus each determinant in the time derivative of $\tilde{F}$ contributes exactly one of the terms $P\left(k_{1}, \ldots, k_{i}-1, \ldots, k_{N} ; t\right)$ on the r.h.s. of (2.1), i.e. (2.15) satisfies the master equation (2.1).

(ii) In order to show that (2.15) satisfies the boundary condition (2.2) we note that according to (2.12) each column in $F$ may be written

$$
\begin{array}{r}
\left(F_{i-1}\left(k_{i}-l_{1}\right), F_{i-2}\left(k_{i}-l_{2}\right), \ldots, F_{i-N}\left(k_{i}-l_{N}\right)\right) \\
=\left(F_{i-1}\left(k_{i}+1-l_{1}\right), F_{i-2}\left(k_{i}+1-l_{2}\right), \ldots, F_{i-N}\left(k_{i}+1-l_{N}\right)\right) \\
+\left(F_{i}\left(k_{i}+1-l_{1}\right), F_{i-1}\left(k_{i}+1-l_{2}\right), \ldots, F_{i+1-N}\left(k_{i}+1-l_{N}\right)\right)
\end{array}
$$


Assume now that $k_{i+1}=k_{i}+1$. This gives for the r.h.s. of (2.2)

$$
\begin{aligned}
& \left|\begin{array}{llll}
F_{0}\left(k_{1}-l_{1} ; t\right) & F_{-1}\left(k_{1}-l_{2} ; t\right) & \ldots & F_{1-N}\left(k_{1}-l_{N} ; t\right) \\
\vdots & \vdots & \vdots & \vdots \\
F_{i-1}\left(k_{i}-l_{1} ; t\right) & F_{i-2}\left(k_{i}-l_{2} ; t\right) & \ldots & F_{i-N}\left(k_{i}-l_{N} ; t\right) \\
F_{i}\left(k_{i+1}-l_{1} ; t\right) & F_{i-1}\left(k_{i+1}-l_{2} ; t\right) & \ldots & F_{i+1-N}\left(k_{i+1}-l_{N} ; t\right) \\
\vdots & \vdots & \vdots & \vdots \\
F_{N-1}\left(k_{N}-l_{1} ; t\right) & F_{N-2}\left(k_{N}-l_{2} ; t\right) & \ldots & F_{0}\left(k_{N}-l_{N} ; t\right)
\end{array}\right| \\
& =\left|\begin{array}{llll}
F_{0}\left(k_{1}-l_{1} ; t\right) & F_{-1}\left(k_{1}-l_{2} ; t\right) & \ldots & F_{1-N}\left(k_{1}-l_{N} ; t\right) \\
\vdots & \vdots & \vdots & \vdots \\
F_{i-1}\left(k_{i}+1-l_{1} ; t\right) & F_{i-2}\left(k_{i}+1-l_{2} ; t\right) & \ldots & F_{i-N}\left(k_{i}+1-l_{N} ; t\right) \\
F_{i}\left(k_{i+1}-l_{1} ; t\right) & F_{i-1}\left(k_{i+1}-l_{2} ; t\right) & \ldots & F_{i+1-N}\left(k_{i+1}-l_{N} ; t\right) \\
\vdots & \vdots & \vdots & \vdots \\
F_{N-1}\left(k_{N}-l_{1} ; t\right) & F_{N-2}\left(k_{N}-l_{2} ; t\right) & \ldots & F_{0}\left(k_{N}-l_{N} ; t\right)
\end{array}\right| \\
& +\left|\begin{array}{llll}
F_{0}\left(k_{1}-l_{1} ; t\right) & F_{-1}\left(k_{1}-l_{2} ; t\right) & \ldots & F_{1-N}\left(k_{1}-l_{N} ; t\right) \\
\vdots & \vdots & \vdots & \vdots \\
F_{i}\left(k_{i}+1-l_{1} ; t\right) & F_{i-1}\left(k_{i}+1-l_{2} ; t\right) & \ldots & F_{i+1-N}\left(k_{i}+1-l_{N} ; t\right) \\
F_{i}\left(k_{i+1}-l_{1} ; t\right) & F_{i-1}\left(k_{i+1}-l_{2} ; t\right) & \ldots & F_{i+1-N}\left(k_{i+1}-l_{N} ; t\right) \\
\vdots & \vdots & \vdots & \vdots \\
F_{N-1}\left(k_{N}-l_{1} ; t\right) & F_{N-2}\left(k_{N}-l_{2} ; t\right) & \ldots & F_{0}\left(k_{N}-l_{N} ; t\right)
\end{array}\right| \\
& =\left|\begin{array}{llll}
F_{0}\left(k_{1}-l_{1} ; t\right) & F_{-1}\left(k_{1}-l_{2} ; t\right) & \ldots & F_{1-N}\left(k_{1}-l_{N} ; t\right) \\
\vdots & \vdots & \vdots & \vdots \\
F_{i-1}\left(k_{i}+1-l_{1} ; t\right) & F_{i-2}\left(k_{i}+1-l_{2} ; t\right) & \ldots & F_{i-N}\left(k_{i}+1-l_{N} ; t\right) \\
F_{i}\left(k_{i+1}-l_{1} ; t\right) & F_{i-1}\left(k_{i+1}-l_{2} ; t\right) & \ldots & F_{i+1-N}\left(k_{i+1}-l_{N} ; t\right) \\
\vdots & \vdots & \vdots & \vdots \\
F_{N-1}\left(k_{N}-l_{1} ; t\right) & F_{N-2}\left(k_{N}-l_{2} ; t\right) & \ldots & F_{0}\left(k_{N}-l_{N} ; t\right)
\end{array}\right|
\end{aligned}
$$

The second determinant in the sum on the r.h.s. of this equation vanishes since two rows are identical. It remains the first determinant in the sum which is equal to the l.h.s. of (2.2).

(iii) It remains to show that (2.15) satisfies the correct initial condition (2.7). We first assume that $k_{1}>l_{1}$. This implies $k_{i}>l_{1}$ since $k_{1}<\ldots<k_{N}$. At $t=0$, all the matrix elements $F_{i 1}=F_{i-1}\left(k_{i}-l_{1} ; 0\right)$ vanish, see (2.14), and hence the determinant vanishes. Now we assume $k_{1}=l_{1}$. Then in the first column $F_{11}=1$, but the other matrix elements $F_{i 1}$ in the first column are still zero, since all $k_{i}$ are larger than $l_{1}$ except $k_{1}$. The determinant of $F$ is therefore equal to the determinant of the matrix $F^{(1)}$ obtained from $F$ by 
omitting the first row and the first column.

In the next step assume now first that $k_{2}>l_{2}$. Repeating the previous step gives $\operatorname{det} F\left(B_{N}, A_{N} ; 0\right)=\delta_{k_{1}, l_{1}} \delta_{k_{2}, l_{2}} \operatorname{det} F^{(2)}$. Iterating this procedure $N$ times finally gives $\operatorname{det} F\left(B_{N}, A_{N} ; 0\right)=\delta_{A_{N}, B_{N}}$.

An integral representation of the terms in the determinant is derived in the next section (3.14).

\section{Bethe ansatz solution}

For a derivation of the solution the master equation (2.1), (2.2), (2.7), we first turn the differential equations (2.1) into an eigenvalue problem by the ansatz $P\left(B_{N} ; t\right)=e^{-\epsilon t} P\left(B_{N}\right)$. In order to solve for the resulting difference equation we follow the strategy employed by Bethe for the solution of the isotropic Heisenberg spin chain [20] and extended by Yang and Yang [21] to the anisotropic spin chain. Rather than using $k_{i}, l_{i}$ for the integer coordinates of the particles we shall use in this section the notation $x_{i}, y_{i} \in Z$. For momentum labels we shall use $p_{i}$.

First we consider $N=1$. The resulting equation is

$$
\epsilon P(x)=-P(x-1)+P(x)
$$

which is readily solved by $P(x)=e^{i p x}$ with $p \in[0,2 \pi)$. This gives for the "energy"

$$
\epsilon_{p}=1-e^{-i p}
$$

and $P(x ; t)=\int_{0}^{2 \pi} d p e^{-\epsilon_{p} t} f(p) e^{i p x}$. The initial condition $P(x ; 0)=\delta_{x, y}$ determines $f(p)=e^{-i p y} /(2 \pi)$ and finally yields

$$
\begin{aligned}
P(x ; t \mid y ; 0) & =\frac{1}{2 \pi} \int_{0}^{2 \pi} d p e^{-\epsilon_{p} t-i p y} e^{i p x} \\
& =\frac{t^{x-y}}{(x-y) !} e^{-t} \\
& =F_{0}(x-y ; t)
\end{aligned}
$$

For $N=2$ one has to solve

$$
\begin{aligned}
\epsilon P\left(x_{1}, x_{2}\right) & =-P\left(x_{1}-1, x_{2}\right)-P\left(x_{1}, x_{2}-1\right)+2 P\left(x_{1}, x_{2}\right) \\
P(x, x) & =P(x, x+1) \quad \forall x
\end{aligned}
$$


The first equation is solved by Bethe's ansatz

$$
P\left(x_{1}, x_{2}\right)=A_{12} e^{i p_{1} x_{1}+i p_{2} x_{2}}+A_{21} e^{i p_{2} x_{1}+i p_{1} x_{2}}
$$

with arbitrary constants $A_{i j}\left(p_{1}, p_{2}\right)$ and gives

$$
\epsilon_{p_{1}, p_{2}}=\epsilon_{p_{1}}+\epsilon_{p_{2}}
$$

The second equation (3.7) fixes the ratio $S_{12} \equiv A_{12} / A_{21}$. Inserting (3.8) gives

$$
S_{12}=-\frac{1-e^{i p_{1}}}{1-e^{i p_{2}}}
$$

The range of values $p_{1}$ and $p_{2}$ may take needs some discussion. In the usual Heisenberg quantum chain one finds a bound state in the twoparticle sector, i.e. a state with complex momenta $p_{1,2}=u \pm i v$. This is a solution for vanishing $A_{12}$ or vanishing $A_{21}$ in which case the wave function decays exponentially in the distance $x_{2}-x_{1}$ (see next Section). Here there is no non-zero $p$ for which either $A_{12}$ or $A_{21}$ vanish and hence no bound state. We conclude that $p_{1}, p_{2} \in[0,2 \pi)$ and $P\left(x_{1}, x_{2} ; t\right)=$ $\int d p_{1} \int d p_{2} e^{-\left(\epsilon_{p_{1}}+\epsilon_{p_{2}}\right) t} f\left(p_{1}, p_{2}\right)\left(e^{i p_{1} x_{1}+i p_{2} x_{2}}+S_{21} e^{i p_{2} x_{1}+i p_{1} x_{2}}\right)$ is the general solution of (2.1) with boundary condition (2.2). (For obvious reasons we define $S_{21} \equiv S_{12}^{-1}$.)

In order to satisfy the initial condition (2.7) one has to determine $f\left(p_{1}, p_{2}\right)$ and discuss the pole resulting from the integration over $S_{21}$. Assuming that the particles were initially at sites $y_{1}, y_{2}$ it turns out that choosing $f\left(p_{1}, p_{2}\right)=$ $e^{-i p_{1} y_{1}-i p_{2} y_{2}}$ and defining the position of the pole in $S_{21}$ by $p_{1} \rightarrow p_{1}+i 0$ gives the correct initial condition $P\left(x_{1}, x_{2} ; 0\right)=\delta_{x_{1}, y_{1}} \delta_{x_{2}, y_{2}}$. This gives

$$
\begin{aligned}
P\left(x_{1}, x_{2} ; t \mid y_{1}, y_{2} ; 0\right)= & \frac{1}{(2 \pi)^{2}} \int_{0}^{2 \pi} d p_{1} \int_{0}^{2 \pi} d p_{2} e^{-\left(\epsilon_{p_{1}}+\epsilon_{p_{2}}\right) t-i p_{1} y_{1}-i p_{2} y_{2}} \\
& \left(e^{i p_{1} x_{1}+i p_{2} x_{2}}-\frac{1-e^{i p_{2}}}{1-e^{i p_{1}}} e^{i p_{2} x_{1}+i p_{1} x_{2}}\right) \\
= & F_{0}\left(x_{1}-y_{1} ; t\right) F_{0}\left(x_{2}-y_{2} ; t\right) \\
& -F_{-1}\left(x_{1}-y_{2} ; t\right) F_{1}\left(x_{2}-y_{1} ; t\right) \\
= & \operatorname{det} F\left(B_{2}, A_{2} ; t\right)
\end{aligned}
$$


with $B_{2}=\left\{x_{1}, x_{2}\right\} \subset \Omega_{2}, A_{2}=\left\{y_{1}, y_{2}\right\} \subset \Omega_{2}$ and the position of the pole in (3.11) defined as discussed above.

In the same way one continues for $N \geq 3$. One constructs a superposition $\Psi=\sum A_{i_{1}, \ldots, i_{N}} \exp \left(i p_{i_{1}} x_{1}+\ldots+i p_{i_{N}} x_{N}\right)$ of $N$-particle plane waves with all $N$ ! possible permutations of the wave numbers $p_{i}$ and with amplitudes $A_{i_{1}, \ldots, i_{N}}$. The ratio of any two amplitudes for plane waves where two momenta $p_{i}, p_{j}$ are interchanged is $S_{i j}$, in the same way as in the two-particle case (3.10). This takes care of the boundary condition (2.2) when (any) two particles are on nearest neighbour sites. The crucial point is that for higher number of particles there are no new constraints from the boundary condition when more than two particles are on adjacent sites. This can be seen by noting that satisfying the boundary condition for any given pair is independent of the coordinates of the remaining particles. So one constructs the Bethe wave function by starting from $e^{i p_{1} x_{1}+\ldots+i p_{N} x_{N}}$ with amplitude $A_{12 \ldots N}=1$ and then performing all possible permutations of the momenta. For each permutation $(i, j) \rightarrow(j, i)$ one multiplies with a factor $S_{j i}$ as in (3.11). The total "energy" $\epsilon$ corresponding to such a wave function is the sum of the single particle energies $\epsilon_{p_{1}, \ldots, p_{N}}=\sum_{i=1}^{N} \epsilon_{p_{i}}$. The initial condition (2.7) determines the overall normalization of the wave function and the position of the poles arising from the integration over the various $S_{i j}$ appearing in the wave function. It is satisfied by the choice $f\left(p_{1}, \ldots, p_{N}\right)=e^{-\left(i p_{1} y_{1}+\ldots i p_{N} y_{N}\right)}$ and by placing the poles in the same way as in the two-particle case, i.e., by setting $p_{i} \rightarrow p_{i}+i 0$ in the denominators.

This construction provides an integral representation of the $N$ ! terms appearing in the determinant (2.15). Therefore the solution of the master equation may be written

$$
\begin{aligned}
P\left(x_{1}, \ldots, x_{N} ; t \mid x_{1}, \ldots, x_{N} ; 0\right)= & \prod_{j=1}^{N} \frac{1}{2 \pi} \int_{0}^{2 \pi} d p_{j} e^{-\epsilon_{p_{j}} t-i p_{j} y_{j}} \times \\
& \Psi_{p_{1}, \ldots, p_{N}}\left(x_{1}, \ldots, x_{N}\right)
\end{aligned}
$$

with the Bethe wave function $\Psi$ as defined above. For three particles it reads

$$
\begin{aligned}
\Psi_{p_{1}, p_{2}, p_{3}}\left(x_{1}, x_{2}, x_{3}\right)= & e^{i p_{1} x_{1}+i p_{2} x_{2}+i p_{3} x_{3}}+S_{21} e^{i p_{2} x_{1}+i p_{1} x_{2}+i p_{3} x_{3}} \\
& +S_{21} S_{31} e^{i p_{2} x_{1}+i p_{3} x_{2}+i p_{1} x_{3}}+S_{21} S_{31} S_{32} e^{i p_{3} x_{1}+i p_{2} x_{2}+i p_{1} x_{3}} \\
& +S_{31} S_{32} e^{i p_{3} x_{1}+i p_{1} x_{2}+i p_{2} x_{3}}+S_{32} e^{i p_{1} x_{1}+i p_{3} x_{2}+i p_{2} x_{3}} \cdot(3.15)
\end{aligned}
$$




\section{The partially asymmetric process}

Using the Bethe ansatz one may also solve for the partially asymmetric process where particles are allowed to move with rate $D_{L}$ to left and with rate $D_{R}$ to the right. The strategy is the same as for the totally asymmetric case discussed in the preceding section. The main difference in the analysis is the occurrence of bound states in addition to the continuum. We shall discuss in some detail only the one- and two-particle systems. This is, in principle, sufficient to construct the general $N$-particle solution.

The case of a single particle can be copied with little modification from the previous section. It is convenient to introduce the asymmetry $q=e^{a}$ and the time scale $D$ by

$$
\begin{aligned}
q & =\sqrt{\frac{D_{R}}{D_{L}}} \\
a & =\ln q \\
D & =\sqrt{D_{R} D_{L}} .
\end{aligned}
$$

After separating the time dependence the master equation reads

$$
\epsilon P(x)=-D_{R} P(x-1)-D_{L} P(x+1)+\left(D_{L}+D_{R}\right) P(x)
$$

which is readily solved by $P(x)=e^{i p x}$ with $p \in[0,2 \pi)$. This gives for the "energy"

$$
\epsilon_{p}=D_{R}\left(1-e^{-i p}\right)+D_{L}\left(1-e^{i p}\right)
$$

and $P(x ; t)=\int_{0}^{2 \pi} d p e^{-\epsilon_{p}} f(p) e^{i p x}$. The initial condition $P(x ; 0)=\delta_{x, y}$ determines $f(p)=e^{-i p y} /(2 \pi)$ and finally yields

$$
\begin{aligned}
P(x ; t \mid y ; 0) & =\frac{1}{2 \pi} \int_{0}^{2 \pi} d p e^{-\epsilon_{p} t-i p y} e^{i p x} \\
& =e^{-\left(q+q^{-1}\right) D t} q^{x-y} I_{x-y}(2 D t)
\end{aligned}
$$

where $I_{n}(\tau)$ is the modified Bessel function. The representation of (4.6) in terms of the Bessel function (4.7) is obtained by an elementary contour integration. It is easy to verify that both expressions (4.6) and (4.7) satisfy the same differential-difference equation with the same initial condition $P(x ; 0 \mid y ; 0)=\delta_{x, y}$. 
Note that the Bessel function diverges asymptotically $\sim e^{2 D t}$ which is not sufficiently fast to cancel the prefactor $e^{-\left(q+q^{-1}\right) D t}$ in (4.7). The interpretation of this observation is that the probability of finding the particle at site $x$ with $x$ kept fixed decays exponentially with an inverse correlation time or "energy gap" $\Delta \epsilon=D\left(q+q^{-1}-2\right)$. However, by going into a comoving frame with velocity $v=D\left(q-q^{-1}\right)=D_{R}-D_{L}$ (see Sec. 5), i.e. by studying the behaviour of the distribution around $x^{\prime}=x+v t$, one finds the usual algebraic, diffusive behaviour.

For $N=2$ one has to solve

$$
\begin{aligned}
\epsilon P\left(x_{1}, x_{2}\right)= & -D_{R}\left(P\left(x_{1}-1, x_{2}\right)+P\left(x_{1}, x_{2}-1\right)-2 P\left(x_{1}, x_{2}\right)\right) \\
& -D_{L}\left(P\left(x_{1}+1, x_{2}\right)+P\left(x_{1}, x_{2}+1\right)-2 P\left(x_{1}, x_{2}\right)\right) \\
P(x, x+1)= & \frac{D_{R} P(x, x)+D_{L} P(x+1, x+1)}{D_{R}+D_{L}} \forall x
\end{aligned}
$$

The first equation is solved by Bethe's ansatz

$$
P\left(x_{1}, x_{2}\right)=A_{12} e^{i p_{1} x_{1}+i p_{2} x_{2}}+A_{21} e^{i p_{2} x_{1}+i p_{1} x_{2}}
$$

with arbitrary constants $A_{i j}\left(p_{1}, p_{2}\right)$ and gives for the two-particle energy

$$
\epsilon_{p_{1}, p_{2}}=\epsilon_{p_{1}}+\epsilon_{p_{2}} .
$$

The second equation (4.9) fixes the ratio $S_{12} \equiv A_{12} / A_{21}$. Inserting (4.10) gives

$$
S_{12}=-\frac{D_{R}+D_{L} e^{i p_{1}+i p_{2}}-\left(D_{R}+D_{L}\right) e^{i p_{1}}}{D_{R}+D_{L} e^{i p_{1}+i p_{2}}-\left(D_{R}+D_{L}\right) e^{i p_{2}}}
$$

which depends only on the momenta $p_{1,2}$ and the asymmetry $q$.

As discussed in the preceding section here one finds besides the continuum $p_{1}, p_{2} \in[0,2 \pi)$ a solution corresponding to a bound state. To see this, we set $p_{1}=u-i(a-v), p_{2}=u-i(a+v)$ with $u, v$ real and $a=\ln q$. Clearly, in order to obtain a wave function which decays exponentially in $x_{2}-x_{1}>0$, either $A_{12}$ or $A_{21}$ must vanish. Choosing

$$
e^{-v}=\frac{\cos u}{\cosh a}<1
$$

gives $A_{12} \equiv D_{R}+D_{L} e^{i p_{1}+i p_{2}}-\left(D_{R}+D_{L}\right) e^{i p_{1}}=0$. The wave function (4.10) 
reduces then to a single expression

$$
P\left(x_{1}, x_{2}\right) \propto q^{x_{1}+x_{2}} e^{i u\left(x_{1}+x_{2}\right)} e^{-v\left(x_{2}-x_{1}\right)} .
$$

Using (4.11) one obtains for the "energy" of this state

$$
\epsilon_{u}=2 D \cosh a\left(1-\frac{\cos ^{2} u}{\cosh ^{2} a}\right)
$$

Thus the bound state has a non-vanishing energy gap $\Delta \epsilon=2 D \sinh ^{2} a / \cosh a$ for any asymmetry $a \neq 0$.

For the determination of the solution of the master equation with initial condition $\delta_{x_{1}, y_{1}} \delta_{x_{2}, y_{2}}$ one proceeds in a way analogous to the totally asymmetric case. First we note that choosing $A_{12}=1$, multiplying the timedependent wave function by $\exp \left(-i p_{1} y_{1}-i p_{2} y_{2}\right)$ and integrating $p_{1}$ and $p_{2}$ from 0 to $2 \pi$ gives, at time $t=0$ the correct initial value $\delta_{x_{1}, y_{1}} \delta_{x_{2}, y_{2}}$ plus a non-vanishing term from the reflected wave proportional to $S_{21}$. This term needs to be cancelled by an appropriate choice of the amplitude of the bound state contribution. This may be determined by using center of mass coordinates $R=x_{1}+x_{2}, R_{0}=y_{1}+y_{2}$ and relative coordinates $r=x_{2}-x_{1}>0$, $r_{0}=y_{2}-y_{1}>0$. Solving first for the $R$-dependence of the master equation (which is trivial) one obtains then a lattice diffusion equation in a single coordinate $r$ with partially absorbing boundary condition. This equation was solved in 22 and one finally finds that

$$
\begin{aligned}
P\left(x_{1}, x_{2} ; t \mid y_{1}, y_{2} ; 0\right)= & \frac{1}{(2 \pi)^{2}} \int_{0}^{2 \pi} d p_{1} \int_{0}^{2 \pi} d p_{2} e^{-\left(\epsilon_{p_{1}}+\epsilon_{p_{2}}\right) t-i p_{1} y_{1}-i p_{2} y_{2}} \times \\
& \left(e^{i p_{1} x_{1}+i p_{2} x_{2}}+S_{21} e^{i p_{2} x_{1}+i p_{1} x_{2}}\right) \\
& +\frac{1}{\pi} \int_{0}^{\pi} d u\left(e^{2 v}-1\right) q^{R-R_{0}} e^{i u\left(R-R_{0}\right)} e^{-v\left(r+r_{0}\right)} e\left(4 . \epsilon^{u} \ddagger 6\right) \\
\equiv & P^{\text {cont }}+P^{\text {bound }}
\end{aligned}
$$

solves the master equation of the partially asymmetric process with two particles and initial condition $P\left(x_{1}, x_{2} ; t \mid y_{1}, y_{2} ; 0\right)=\delta_{x_{1}, y_{1}} \delta_{x_{2}, y_{2}}$. The first piece $P^{\text {cont }}$ in the sum is the contribution from the continuum of states (4.10), whereas in the second piece $P^{\text {bound }}$ one recognizes the contribution from the 
bound state. Using the identity

$$
\mu^{-n} I_{n}(2 \tau)=\frac{1}{2 \pi} \int_{0}^{2 \pi} d p e^{i p n+\left(\mu e^{i p}+\mu^{-1} e^{-i p}\right) \tau}
$$

and expanding the denominator of $S_{21}$ in a geometric series in $z\left(p_{1}, p_{2}\right)=$ $\left(D_{R} e^{-i p_{2}}+D_{L} e^{i p_{1}}\right) /\left(D_{R}+D_{L}\right)$ one may rewrite (4.16) in terms of a sum of products of two modified Bessel functions. With (4.18) the bound state contribution takes the form

$$
P^{\text {bound }}=\frac{1}{\pi} \int_{0}^{\pi} d u e^{i u\left(R-R_{0}\right)}\left(1-\xi^{2}\right) \xi^{r+r_{0}-2} e^{-\left(1-\xi^{2}\right)\left(D_{R}+D_{L}\right) t}
$$

where $\xi(u)=z(u,-u)$.

The general $N$-particle problem is solved by the Bethe ansatz for $N$ particles and by determining the various bound state contributions. A way of determining the contributions from the bound states in the general case is by an appropriate contour integration in the complex $k_{i}$ planes which includes the poles of the reflection coefficients $S_{i j}$. These poles give rise to the bound state contributions in a general $N$-particle problem.

\section{Diffusion of two particles}

Here we want to study how the exclusion interaction affects the diffusion of two particles. In order to get an understanding of what is happening we put the particles at time $t=0$ on lattice sites $y_{1}=-1$ and $y_{2}=1$ and we study the moments of the density distribution at time $t$. This describes the diffusive broadening of an initially spatially concentrated density.

We introduce expectation value $\left\langle n_{x}\right\rangle$ which is the probability of finding a particle on site $x$ at time $t$. The moments of this density distribution may be obtained from the Fourier transform $\hat{\rho}(q)=\sum_{x} e^{-i q x}\left\langle n_{x}\right\rangle$ by taking derivatives w.r.t $q$. Here we are interested in

$$
\begin{aligned}
N & =\hat{\rho}(0)=2 \\
\langle X\rangle & =\frac{i}{N} \hat{\rho}^{\prime}(0)=\frac{1}{2} \sum_{x} x\left\langle n_{x}\right\rangle \\
\left\langle X^{2}\right\rangle & =-\frac{1}{N} \hat{\rho}^{\prime \prime}(0)=\frac{1}{2} \sum_{x} x^{2}\left\langle n_{x}\right\rangle
\end{aligned}
$$


from which we shall calculate the asymptotic drift velocity $v$ and the asymptotic collective diffusion constant $\Delta$ defined by

$$
\begin{aligned}
v & =\lim _{t \rightarrow \infty} \frac{d}{d t}\langle X\rangle \\
\Delta & =\lim _{t \rightarrow \infty} \frac{d}{d t}\left(\left\langle X^{2}\right\rangle-\langle X\rangle^{2}\right)
\end{aligned}
$$

It may be useful to remind the reader of what these quantities are in case of non-interacting particles. This allows for a comparison of the interacting and the non-interacting system. For a single particle or for two non-interacting particles the density satisfies the diffusion equation

$$
\frac{d}{d t}\left\langle n_{x}\right\rangle=D_{R}\left\langle n_{x-1}\right\rangle+D_{L}\left\langle n_{x+1}\right\rangle-\left(D_{R}+D_{L}\right)\left\langle n_{x}\right\rangle
$$

Integrating this equation gives after a short calculation

$$
\begin{aligned}
v & =D_{R}-D_{L} \\
\Delta & =D_{R}+D_{L}
\end{aligned}
$$

which is trivial in the sense that this is just a way of defining the driven non-interacting process. The non-trivial point is the determination of these quantities for the system with exclusion interaction.

First we note that for the ASEP the density satisfies the continuity equation

$$
\frac{d}{d t}\left\langle n_{x}\right\rangle=\left\langle j_{x-1}\right\rangle-\left\langle j_{x}\right\rangle
$$

with the current

$$
\left\langle j_{x}\right\rangle=D_{R}\left\langle n_{x}\left(1-n_{x+1}\right)\right\rangle-D_{L}\left\langle\left(1-n_{x}\right) n_{x+1}\right\rangle
$$

Thus one gets $v=\sum_{x}\left\langle j_{x}\right\rangle / 2=\left(D_{R}-D_{L}\right) \sum_{x}\left(\left\langle n_{x}\right\rangle-\left\langle n_{x} n_{x+1}\right\rangle\right) / 2=$ $\left(D_{R}-D_{L}\right)\left(1-\sum_{x}\left\langle n_{x} n_{x+1}\right\rangle / 2\right)$. Using (4.9) one may write $\sum_{x}\left\langle n_{x} n_{x+1}\right\rangle=$ $\sum_{x} P(x, x+1 ; t)=\sum_{x}\left(D_{R} P(x, x ; t)+D_{L} P(x+1, x+1 ; t)\right) /\left(D_{R}+D_{L}\right)=$ $\sum_{x} P(x, x ; t)$. Thus with the initial condition considered above one gets

$$
\langle X\rangle=\left(D_{R}-D_{L}\right)\left(t-\frac{1}{2} \int_{0}^{t} d \tau \sum_{x} P(x, x ; \tau \mid-1,1 ; 0)\right) .
$$


Using the continuity equation the diffusion constant may be written $\Delta=\sum_{x}(x+1 / 2)\left\langle j_{x}\right\rangle-2 v\langle X\rangle=v+2 D_{L}+2\left(D_{R}-D_{L}-v\right)\langle X\rangle-\left(D_{R}-\right.$ $\left.D_{L}\right) \sum_{x} x\left\langle n_{x} n_{x+1}\right\rangle$. Now (5.11) and (4.9) lead to

$$
\begin{aligned}
\Delta= & \lim _{t \rightarrow \infty}\left\{D_{R}+D_{L}+\right. \\
& \left(D_{R}-D_{L}\right)^{2} \sum_{x} P(x, x ; t \mid-1,1 ; 0)\left[t-\frac{1}{2} \int_{0}^{t} d \tau \sum_{x} P(x, x ; \tau \mid-1,1 ; 0)\right] \\
& \left.-\left(D_{R}-D_{L}\right)\left[\sum_{x}\left(x-\frac{D_{R}-D_{L}}{2\left(D_{R}+D_{L}\right)}\right) P(x, x ; t \mid-1,1 ; 0)\right]\right\}
\end{aligned}
$$

Therefore in order to determine $v$ and $D$ and has to calculate $P_{0}=$ $\sum_{x} P(x, x ; t \mid-1,1 ; 0)$ and $P_{1}=\sum_{x} x P(x, x ; t \mid-1,1 ; 0)$. Each of these quantities can be split into three contributions arising from the two contributions from the continuum and the bound state contribution in (4.16).

There is no contribution from the bound state to $\sum_{x} P(x, x ; t \mid-1,1 ; 0)$ and defining $\tau=2\left(D_{R}+D_{L}\right) t$ one finds

$$
P_{0}=e^{-\tau}\left(I_{2}(\tau)+I_{1}(\tau)\right)
$$

where the term proportional to $I_{1}$ is the term arising from the reflected wave proportional to $S_{21}$.

The calculation of $P_{1}$ is slightly more involved, but still straightforward, and gives

$$
\begin{aligned}
P_{1}= & \frac{\tau}{2\left(D_{R}+D_{L}\right)} e^{-\tau}\left(D_{R} I_{3}-D_{L} I_{1}\right)+e^{-\tau} I_{2} \\
& +\frac{\tau}{2\left(D_{R}+D_{L}\right)} e^{-\tau}\left(D_{R} I_{2}-D_{L} I_{0}\right)+\frac{D_{R}-D_{L}}{2\left(D_{R}+D_{L}\right)}\left(1+e^{-\tau} I_{0}\right)+\frac{D_{R}}{D_{R}+D_{L}} e^{-\tau} I_{1} \\
& -\frac{D_{R}-D_{L}}{D_{R}+D_{L}}
\end{aligned}
$$

where the last piece in the sum comes from the bound state and the arguments of the Bessel functions are all $\tau$.

Putting everything together and taking the limit $t \rightarrow \infty$ in (5.12) finally 
yields

$$
\begin{aligned}
v & =D_{R}-D_{L} \\
\Delta & =D_{R}+D_{L}+\frac{\left(D_{R}-D_{L}\right)^{2}}{D_{R}+D_{L}}\left(\frac{1}{2}-\frac{1}{\pi}\right) .
\end{aligned}
$$

On comparison with the results (5.7), (5.8) for non-interacting particles one notices that the exclusion interaction alone does not change the collective two-particle diffusion constant. In the undriven system one has $\Delta=D_{R}+D_{L}$ as in the non-interacting system. In the presence of the drift, however, $\Delta$ increases to the value (5.16). This result was obtained for particles placed initially at sites $x_{1,2}=-1,1$. It is however valid for any (finite) initial separation $r_{0}$. After a time $t_{0} \gg r_{0}^{2}$ the details of the initial condition are washed out.

\section{Conclusions}

The main result of this paper is the solution (2.15) of the master equation for the asymmetric simple exclusion process. This solution allows for a complete description of a system of finitely many particles. As a simple example we have investigated the collective diffusion of two single particles. We found that the diffusive broadening of the density profile in the driven system (5.16) is faster than both in the undriven and in the non-interacting case.

The solution of the master equation may also be used for the analysis of quantities in systems with finite density. Consider e.g. a system with constant non-zero density $\rho$ with a local inhomogeneity such as a lattice site $y$ where initially the density is $\left\langle n_{y}\right\rangle=1$. In such a situation it would be interesting to study the time evolution of the density profile (which gives the dynamical structure function) or the temporal behaviour of density correlations. Using the exact solution one can obtain an exact expansion of these quantities in powers of $\rho$ where the $n^{t h}$ power is obtained by solving the $n$ particle problem. This can be seen as follows: Suppose one wants to calculate the time-dependent density profile $\rho_{x}(t)=\left\langle n_{x}(t)\right\rangle$ up to second order in the background density $\rho$. The time derivative of the two-point correlation function involves a three-point correlator which is of order $\rho^{3}$ and which therefore may be neglected in the desired second order approximation. Leaving the three-point correlator out results in a differential-difference equation for the two-point correlator which is identical to the two-particle master equation (2.1) with boundary condition (2.2). Thus one can calculate $\left\langle n_{x} n_{y}\right\rangle$ and then by summing up two-point correlators one gets $\left\langle n_{x}\right\rangle$ up to order $\rho^{2}$. For

a third order approximation one considers the three-point correlation func- 
tion. If one neglects fourth order correlators, it satisfies the three-particle master equation. Summing up three-point correlators yields $\left\langle n_{x}\right\rangle$ up to order $\rho^{3}$.

A by-product of Sec. 5 is the explicit solution of the two-particle problem for the symmetric exclusion process. It is interesting to recall that in the symmetric case $D_{R}=D_{L}$ the conditional probability $P\left(x_{1}, x_{2} ; t \mid y_{1}, y_{2} ; 0\right)$ determines not only the behaviour of the two-particle system, but also the behaviour of various time-dependent density-density correlation functions in $N$-particle systems, viz. the equal-time two-point correlator $\left\langle n_{x_{1}}(t) n_{x_{2}}(t)\right\rangle$ for an arbitrary initial state [3], the two-time correlator $\left\langle n_{x_{1}}\left(t_{1}\right) n_{x_{2}}\left(t_{2}\right)\right\rangle$ for an arbitrary initial state [23] and the (time-translationally invariant) fourpoint correlator $\left\langle n_{x_{1}}\left(t_{1}\right) n_{x_{2}}\left(t_{2}\right) n_{x_{3}}\left(t_{3}\right) n_{x_{4}}\left(t_{4}\right)\right\rangle$ averaged over the stationary distribution [23]. The contribution of the bound state to pair diffusion was discussed in [24]. It would be interesting to investigate in detail how the bound state effects the behaviour of these correlators in the various spacetime regimes of the symmetric diffusion process.

One puzzling problem is the behaviour of the ASEP in the presence of a blockage [25, 26, 27], i.e., a bond in the lattice where particles hop with rate $r \neq 1$. Numerical and analytical studies seem to indicate that the steady state current in a finite, half-filled system approaches its maximal value $j_{\max }=1 / 4$ already at a surprisingly small defect hopping rate $r \lesssim 0.8$ [27, 28, 29]. This raises the question of a non-analyticity in the current $j(r)$ for $r<1$ which only an exact calculation of the steady state current can resolve. A perturbative expansion in $r$ has been performed up to sixth order using computer algebra [28]. Each coefficient in the expansion is a rational number with numerators and denominators rapidly increasing with the order. Many non-trivial exact steady state properties of exclusion processes have been obtained by exact calculation for e.g. small system sizes, then guessing the general structure, and finally proving that the exact expressions obtained in this way are correct [10, 11, 30, 31, 32, 33. Unfortunately, applying this strategy to the perturbative coefficients obtained in [28] seems hopeless. However, a systematic perturbative expansion of the current may be performed using the solution (2.15) of the master equation where the $n^{\text {th }}$ order in $r$ is obtained by the solution of the $n$-particle problem. This gives the coefficients of $r^{n}$ as a sum of $n$ ! fractions with slowly increasing denominators and numerators rather than the single fractions given in Ref. [28]. Therefore there is some hope that one might be able to guess a pattern in this sequence of fractions. Clearly this is a somewhat speculative suggestion which very well may turn out to be useless. But it seems worth trying to obtain exact results in this way. 


\section{Acknowledgments}

The author would like to thank J. Lebowitz, E. Speer and R. Stinchcombe for stimulating discussions and the Department of Mathematics, Rutgers University, where part of this work was done, for kind hospitality.

\section{References}

[1] B. Schmittmann and R.K.P. Zia, Statistical mechanics of driven diffusive systems, in Phase Transitions and Critical Phenomena, eds. C Domb and J. Lebowitz (Academic, London, 1995), Vol. 17.

[2] C.T. MacDonald, J.H. Gibbs and A.C. Pipkin, Biopolymers 6, 1 (1968).

[3] F. Spitzer, Adv. Math. $\underline{5}, 246$ (1970).

[4] T. Ligget, Interacting Particle Systems, New York, Springer Verlag (1985)

[5] J. Krug and H. Spohn, in: Solids far from Equilibrium, ed. C. Godreche, (Cambridge, Cambridge University Press, 1991) and references therein.

[6] J.M. Burgers, The nonlinear diffusion equation (Riedel, Boston, 1974).

[7] B. Derrida, S. A. Janowsky, J. L. Lebowitz and E. R. Speer, Europhys. Lett. 22, 651 (1993).

[8] P.A. Ferrari and L.R.G. Fontes, Probab. Theory Relat. Fields 99, 305 (1994).

[9] K. Nagel, Phys. Rev. E 53, 4655 (1996).

[10] G. Schütz and E. Domany, J. Stat. Phys. 72, 277 (1993).

[11] B. Derrida, M. R. Evans, V. Hakim and V. Pasquier, J. Phys. A 26, 1493 (1993).

[12] S. Sandow, Phys. Rev. E 50, 2660 (1994).

[13] F.H.L. Essler and V. Rittenberg, J. Phys. A 29, 3375 (1996).

[14] H. Rost, Z. Wahrsch. verw. Gebiete 58, 41 (1982).

[15] L.-H. Gwa and H. Spohn, Phys. Rev. A, 46, 844 (1992).

[16] B. Derrida, M. R. Evans, in: Nonequilibrium Statistical Mechanics in One Dimension, ed. V. Privman, (Cambridge, Cambridge University Press, in press). 
[17] S. Sandow and G. Schütz, Europhys. Lett. 26, 7 (1994).

[18] D. Kim, Phys. Rev. E 52, 3512 (1995).

[19] G.M. Schütz, Duality Relations for Asymmetric Exclusion Processes, to appear in J. Stat. Phys. (1997)

[20] H. Bethe, Z. Phys. 71, 205 (1931).

[21] C.N. Yang and C.P. Yang, Phys. Rev. 150, 327 (1966).

[22] R.B. Stinchcombe and G.M. Schütz, Europhys. Lett. 29, 663 (1995).

[23] G. Schütz and S. Sandow, Phys. Rev. E 49, 2726 (1994).

[24] W. Dieterich and I. Peschel, J. Phys. C 16, 3841 (1983).

[25] D.E. Wolf and L.-H. Tang, Phys. Rev. Lett., 65, 1591 (1990).

[26] L.-H. Tang and I.F. Lyuksyutov, Phys. Rev. Lett., 71, 2745 (1993).

[27] S.A. Janowsky and J.L. Lebowitz, Phys. Rev. A 45, 618 (1992).

[28] S.A. Janowsky and J.L. Lebowitz, J. Stat. Phys. 77, 35 (1994).

[29] E. Speer, private communication.

[30] B. Derrida, E. Domany and D. Mukamel, J. Stat. Phys. 69, 667 (1992).

[31] G. Schütz, J. Stat. Phys. 71, 471 (1993).

[32] G. Schütz, Phys. Rev. E 47, 4265 (1993).

[33] H. Hinrichsen, J. Phys. A 29, 3659 (1996). 